\title{
Quantification of white matter fibre pathways disruption in frontal transcortical approach to the lateral ventricle or the interventricular foramen in diffusion tensor tractography
}

\author{
T. Szmuda ${ }^{1}$, P. Słoniewski ${ }^{1}$, M. Szmuda² ${ }^{2}$ P.M. Waszak ${ }^{3}$, A. Starzyńska ${ }^{4}$ \\ ${ }^{1}$ Neurosurgery Department, Medical University of Gdansk, Poland \\ ${ }^{2}$ Developmental Neurology Department, Medical University of Gdansk, Poland \\ ${ }^{3}$ Students Scientific Association of Neurosurgery Department, Medical University of Gdansk, Poland \\ ${ }^{4}$ Department of Maxillofacial and Oral Surgery, Medical University of Gdansk, Poland
}

[Received 10 June 2013; Accepted 31 August 2013]

Pathologies occupying the interventricular foramen (foramen of Monro - FM) or the anterior part of lateral ventricle (LV) are accessed by the transcortical or transcallosal route. As severing of rostral corpus callosum has been deemed inferior to cortical incision, the approaches through various points of frontal lobe have been developed. Superior (F1), middle (F2) frontal gyrus or occasionally superior frontal sulcus are used as an entry of neurosurgical corridor. In spite of the fact that every approach to LV or FM causes its characteristic irreversible damage to white matter, to date all of transcortical routes are regarded as equivalent. The current study compared the damage of main neural bundles between virtual trans-F1 and trans-F2 corridors by means of diffusion tensor tractography method (DTT) in 11 magnetic resonance imaging (MRI) exams from clinical series (22 hemispheres, regardless of dominance). Corpus callosum, cingulum, subdivisions I and II of superior longitudinal fasciculus (SLF I and SLF II), corticoreticular as well as pyramidal tracts crossing both approaches were subjected to surgical violation. Both approaches served a similar total number of fibres ( 0.94 to $\left.1.78\left[\times 10^{3}\right]\right)$. Trans-F1 route caused significantly greater damage of total white matter volume (F1: 8.26 vs. F2: $7.16 \mathrm{~mL}$ ), percentage of SLF I fibres (F1: $78.6 \%$ vs. F2: $28.6 \%$ ) and cingulum ( $F 1: 49.4 \%$ vs. F2: $10.6 \%$ ), whereas trans-F2 route interrupted more corticoreticular fibres (F1: $4.5 \%$ vs. F2: $30.7 \%$ ). Pyramidal tract (F1: $0.6 \%$ vs. F2: 1.3\%) and SLF II (F1: $15.9 \%$ vs. F2: $26.2 \%$ ) were marginally more vulnerable in case of the access via middle frontal gyrus. Both approaches destroyed $7 \%$ of callosal fibres. Summarising the above DTT findings, trans-F2 route disrupted a greater number of fibres from eloquent neural bundles (SLF II, pyramidal and corticoreticular tracts), therefore is regarded as inferior to trans-F1 one. Due to lack of up-to-date guidelines with recommendations of the approaches to LV or FM, an individual preoperative planning based on DTT should precede a surgery. (Folia Morphol 2014; 73, 2: 129-138)

Key words: neural fibres, frontal lobe, superior frontal gyrus, middle frontal gyrus, diffusion tensor imaging, neurosurgical approach 


\section{INTRODUCTION}

The body of lateral ventricle (LV) extends rostrally into the frontal lobe, composing the anterior (frontal) horn. The rostral part of the greatest chamber of the brain connects ventrally with $3^{\text {rd }}$ ventricle by the interventricular foramen (foramen of Monro - FM) [36]. Numerous neural fibres (corpus callosum, subdivisions of superior longitudinal fasciculus SLF I and II, corticoreticular pathway, pyramidal tract, short association fibres) and brain cortex of frontal lobe (Brodmann areas 6, 8, 9, supplementary motor cortex - SMA and pre-SMA - preSMA) cover superior rostral part of LV. Therefore, historical or contemporary surgical route to LV and FM, to some extent involves vital fibres.

A variety of neurosurgical approaches to the pathologies originating from these locations has been developed over the years. Walter Dandy was the first who described anterior transcortical exposure of tumours occupying LV or FM [40]. Then, an extended frontal lobe incision (oval resection or transverse section on non-dominant side) resulted in frequent occurrence of post-operative seizures [13].
Contemporarily, 2 different, nonetheless equivalent, transcortical trajectories lead to anterior horn of LV or FM: one passes through the upper frontal gyrus (trans-F1) and the second through the middle frontal lobe (trans-F2) [14, 52]. Others consider transcallosal interhemispheric route as principal $[53,55]$. To date, a neurosurgeon's preference dictated the choice of the above approaches, as no guidelines regarding this issue were reported. Moreover, modern keyhole approaches concern relatively small cortical incision and thus a funnel-like operating corridor. The above-mentioned strategy has decreased the violation of brain cortex, but parallel to the expanding of subcortical disruption (Fig. 1).

A variety of complications related to access to $L V$ or FM can occur. Increased seizure occurrence rate is related to the transcallosal interhemispheric approach, where paradoxically anatomic considerations would be conductive to that route [5]. However, a great callosal incision, coagulation of medial draining veins on routine basis and unintended pericallosal artery injury are regarded as negative aspects of transcallosal access in some reports $[23,24]$. In

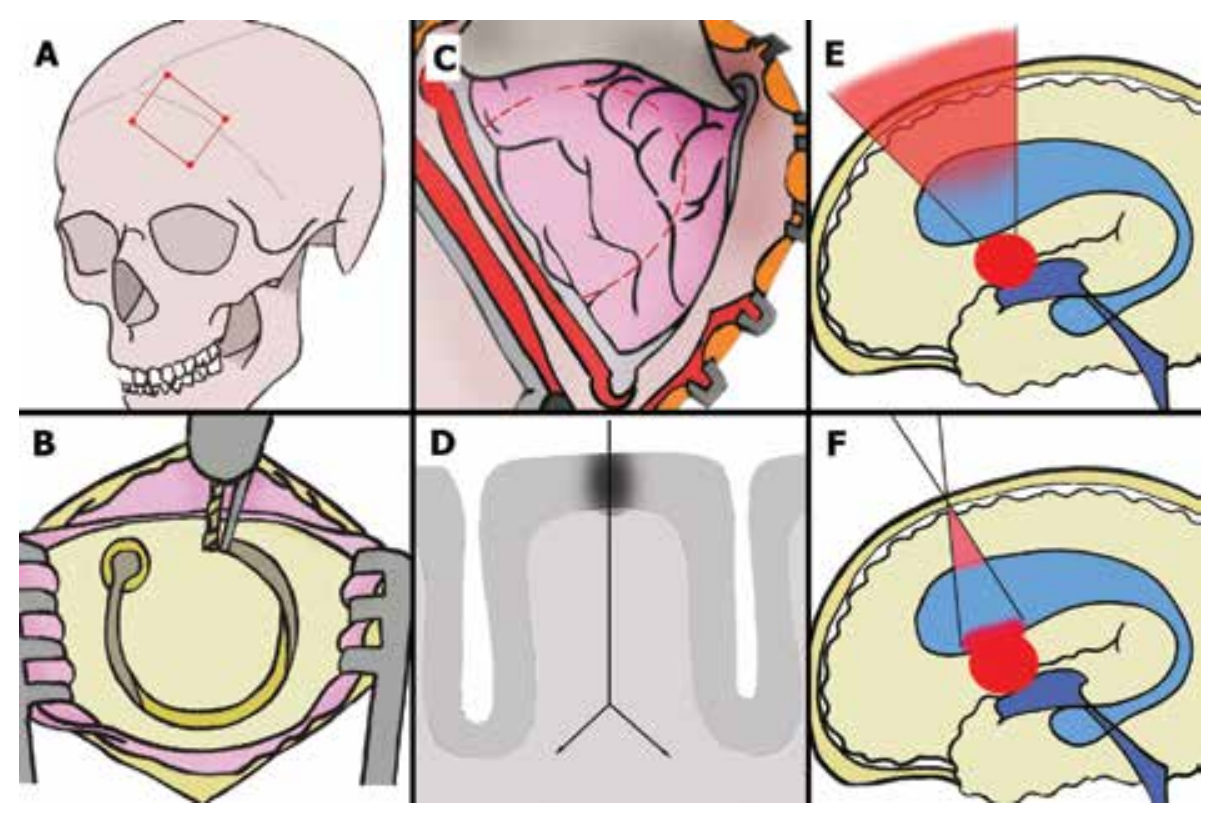

Figure 1. The placement of craniotomy, cortical incision and the idea of keyhole approach to lateral ventricle and the interventricular foramen; A. The historical method of the frontal approach to the $3^{\text {rd }}$ ventricle lesions. This exposure was used in an access to the ventricular system in hydrocephalic state. Primarily it was described by Apuzzo [4]; B. Contemporary craniotomy is limited to 1 burr hole (3 cm in diameter). Its proper placement and range are planned preoperatively using navigation systems; C, D. Most of the ventricular lesions are deep-seated. Vast cortical and white matter dissections, supported by an extended retraction of the frontal lobe, were related to high mortality and morbidity when a historical transcortical approach was applied (C). On the other hand, that type of the neurosurgical approach can provide an opportunity to visualise large ventricular lesions, which facilitate its removal. Contemporary small cortical incision and transgyral white matter dissection (D) preserve more vital neural structures than the historical approach to the ventricles; $\mathbf{E}$, F. The keyhole transcortical approach (F) is used for the access to the $3^{\text {rd }}$ ventricle. a minimal craniotomy and limited frontal lobe incision are required. However, the keyhole access produces similar destruction of white matter pathways as the standard approach (E). 
transcortical route, up to $20 \%$ of patients may develop hemiparesis, which is attributed to the violation of centrum semiovale $[3,15,39]$. Memory deficits occur after the damage of nucleus caudatus or thalamocortical fibres [20]. Alexia, agraphia or spatial neglect are another complications of transcortical approach caused by an interruption of subcortical SLF $[44,45]$.

The application of the preoperative planning, using the preoperative magnetic resonance imaging (MRI) and the intraoperative navigation systems, were milestones in neurosurgery. It finally facilitated the optimum anatomical placement of a craniotomy, cortical incision, as well as exclusive exposing a targeted region [48]. Since further introduction of the in vivo visualisation and localisation of white matter pathways by diffusion tensor tractography method (DTT), the strategy of either targeted craniotomy or tumour resection has changed [12]. DTT is the MRI-based, non-invasive method that measures directional anisotropy of water [7]. By following the directions of diffusion (diffusion tensor imaging DTI), DTT estimates the trajectories of fibre tracts and displays a detailed map of their configuration as a portrayal of anatomical structures 3-dimensionally. That method is limited to gross fibre architecture. The whole planning can be then incorporated into a navigation system. Generally, DTT facilitates the resection of lesions infiltrating eloquent areas concerning language and motor function, and is also preclinically used in patients with neurological or psychiatric diseases [31, 43].

Two general principles of every transcortical approach to LV or FM, namely craniotomy anterior to coronal suture and access through wider LV, argue with recent technological capabilities [40]. To the best of our knowledge, neither quantification of white matter pathways in any frontal approach nor comparison of incised fibres between trans-F1 nor trans-F2 route have been published yet. The presented study, which applies modern neural fibres visualisation technique, seems to be of interest to researchers in the neuroscience field and neurosurgeons alike.

\section{MATERIALS AND METHODS}

MRI of the brain (as follows: T1, T2 and DTI with single-shot echo-planar imaging) were acquired using a 20-channel Head/Neck coil on a 1.5 T Siemens Magnetom Aera scanner (Erlangen, Germany). Three repetitions of 20 non-collinear diffusion gradient directions for contiguous slices were used. All slices were acquired parallel to the anterior commissureposterior commissure line. Imaging parameters of DTI acquisition: $5.0 \mathrm{~mm}$ slice thickness, $128 \times 128$ matrix, $240 \mathrm{~mm} \times 240 \mathrm{~mm}$ field of view, $\mathrm{b}=1000 \mathrm{~s} / \mathrm{mm}^{2}$, repetition time $=3500 \mathrm{~ms}$, echo time $=83.0 \mathrm{~ms}$ and weak fat suppression, were used in the study. DTI output volumes of tensors were computed from the derived tensor information by means of Fractional Anisotropy (FA), Directionally Encoded Colours (DEC), Apparent Diffusion Coefficient (ADC) and Mean Diffusivity (MD). Output volumes were co-registered with $\mathrm{T} 1$ exam as an anatomical reference. Fibre tracking was performed using a probabilistic method of multi-fibre model. FA level of 0.2 was set, seed density was held at 5.0, minimal fibre length was set to $20 \mathrm{~mm}$, curvature threshold of 0.2 , step length of $0.5 \mathrm{~mm}$ and maximal directional change of fibres was chosen between 45 and 50 degrees. Each neural tract group (neural bundle), which passed the hypothetic trans-F1 and trans-F2 corridor to LV, was determined by seeding start and end regions of interest in a specific location. Corpus callosum, cingulum, pyramidal and corticoreticular tracts, short association fibres, SLF I, as well as SLF II were respectively segmented by both selecting and excluding different anatomical landmarks according to the methods proposed in previous reports (for corpus callosum [1], for cingulum, for pyramidal tract [9], for short association fibres, for SLF I [27], for SLF II [17]). FA, ADC, total crossing quantity and volume of fibres were calculated for hypothetically devastating trans-F1 and trans-F2 approaches. Region of interest (ROI) was set at the entire neurosurgical approach; the mean value of separate measurements of diffusion was drawn.

The analysis of specific neural bundle damage caused by an approach concerned the analysis of quantity and volume of fibres. All of DTI/DDT measurements and post-processing were performed, refined and depicted using StealthViz surgical planning software (Medtronic Inc., Minneapolis, USA) and TrackVis (www.trackvis.org, Department of Radiology, Massachusetts General Hospital, USA). StealthViz was used in case of the following calculations: number and length of fibres, FA, and ADC mean value. Total volume of white matter as well as total volume of fibres disrupted by trans-F1 or trans-F2 approach were calculated by means of TrackVis software. Only StealthViz is approved and registered for medical use by Food and Drug Administration. 


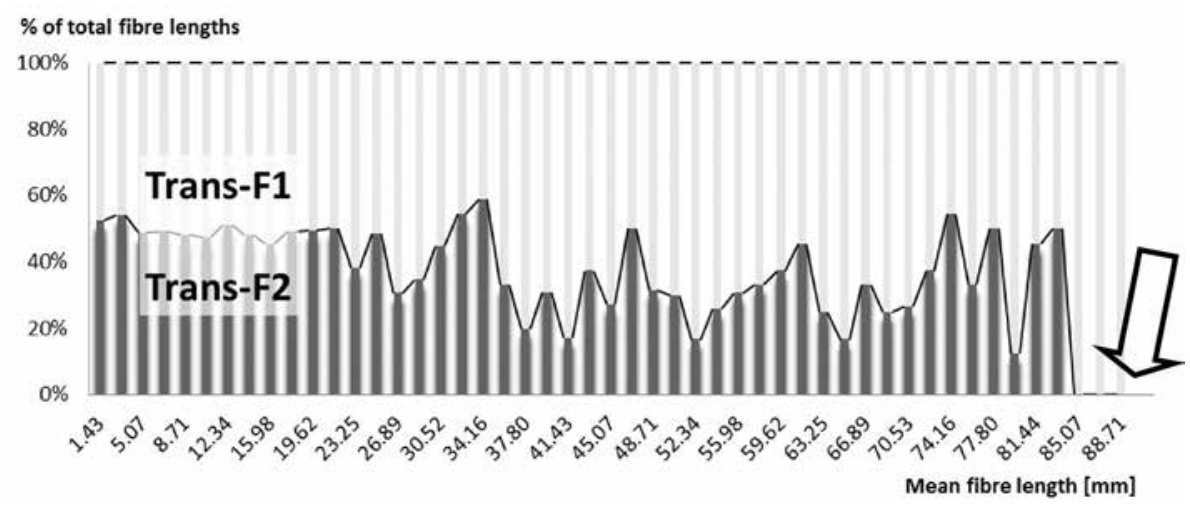

Figure 2. The $100 \%$ column chart depicting relative proportion between trans-F1 and trans-F2 approach in terms of violated diffusion tensor imaging fibre lengths. The cumulative proportion of a single stacked fibre length totals $100 \%$. The longest fibres that were cut always belonged to the trans-F1 corridor (arrow).

MRI exams were performed in 11 patients from clinical series ( 7 males and 5 females), which allowed the analysis of 22 hemispheres. The age of subjects varied between 36 and 76 (mean age was 55.6; standard deviation [SD] $=11.2$ ). Volumetric asymmetry and symmetry coefficients between hemispheres were not calculated; hemisphere dominance was not evaluated. Nine of the presented MRIs were of brains with tumours in other locations of the brains that were not affecting neural fibres assessment of hypothetical frontal transcortical approach to LV or FM. One MRI showed tumour located in the anterior part of the body of LV, another in FM. In those patients harbouring ventricular tumour, the evaluation of white matter fibre bundles was validated by the clinical use. The current study data has been retrospectively collected in years 2012 and 2013 at the Neurosurgery Department. Due to the observational character of our study and blinded patients' data, the local Ethical Committee approval was not obligatory.

Descriptive statistics, including mean value, SD and range were included. An independent t-test or Mann-Whitney test (for not normally distributed data) was applied to determine the variances between 2 analysed approaches. Statistica v.10.0 (StatSoft, Tulsa Inc., USA) was used for statistical analysis, with the significance level $(p)$ set at 0.05 .

\section{RESULTS}

The extension of white matter disruption, measured in terms of total volumes, was greater in case of trans-F1 approach. Total volume of all fibres, of which connectivity was potentially destroyed by a corridor, did not differ between the approaches. Mean ADC of all fibres crossing the ROI (region of interest) of trans-F1 occurred significantly higher than the ADC of trans-F2. Similarly, mean FA of all fibres was increased if ROI was set on trans-F2, but the comparison occurred insignificant. The approach through superior frontal gyrus destroyed insignificantly longer DTI fibres than trans-F2 approach. Fibres longer than $83.3 \mathrm{~mm}$ did not cross trans-F2 trajectory at all, whereas there were 11 DTI fibres on average in the area of trans-F1 corridor (Fig. 2, Table 1).

The qualitative evaluation of white matter bundles crossing frontal transcortical approaches revealed that the access through either superior or medial frontal gyrus at least partially sectioned following tracts: division I and II of SLF, 2 sub-volumes of rostral corpus callosum (CC1/CC2 [22]), pyramidal and corticoreticular tracts. The presented result was consistently noted in every analysed hemisphere (22 of $22 ; 100 \%)$. The exception was cingulum, which was not violated during trans-F2 approach in 20 of the 22 investigated cases (90.9\%). The disruption of short association fibres depended on a specific gyrus, where the cortical incision was hypothetically set off. The constraint regarding different short association fibres of superior and middle frontal gyri prevented their qualitative comparison (Fig. 3).

Total number of fibres composing a whole bundle was quantified. A part of disrupted SLF I/II, pyramidal and corticoreticular tracts, cingulum, and corpus callosum were outlined during both frontal transcortical approaches as well as its fibres were quantified. The percentage of fibres of a given neural bundle that was cut during trans-F1 and trans-F2 approach was calculated (Fig. 4). 
Table 1. Total volume of white matter; number, length and volume of diffusion tensor imaging (DTI) fibres; fractional anisotropy (FA) and apparent diffusion coefficient (ADC) of DTI fibres, which were destroyed by a hypothetical trans-F1 and trans-F2 approach

\begin{tabular}{lcccccc}
\hline $\begin{array}{l}\text { Hypothetical } \\
\text { approach }\end{array}$ & $\begin{array}{c}\text { Total volume of } \\
\text { white matter } \\
\text { damage by the } \\
\text { surgery [mL] }\end{array}$ & $\begin{array}{c}\text { Total fibre track } \\
\text { volume being } \\
\text { disrupted }[\mathbf{m L}]\end{array}$ & $\begin{array}{c}\text { Total number of } \\
\text { DTI fibres being } \\
\text { disrupted }\left[\times \mathbf{1 0}^{3}\right]\end{array}$ & $\begin{array}{c}\text { Length of all DTI } \\
\text { fibres crossing } \\
\text { the approach [mm] }\end{array}$ & $\begin{array}{c}\text { FA of all fibres } \\
\text { crossing the } \\
\text { approach } \\
\text { [scalar value 0-1] }\end{array}$ & $\begin{array}{c}\text { ADC of all fibres } \\
\text { crossing the } \\
\text { approach }\left[\boldsymbol{\mu m} \mathbf{m}^{2} / \mathbf{s}\right]\end{array}$ \\
\hline Trans-F1 & $8.26 \pm 1.24$ & $86.2 \pm 17.7$ & $1.17 \pm 0.35$ & $24.30 \pm 21.06$ & $0.41 \pm 0.13$ & $897.3 \pm 181.8$ \\
& $(6.15-10.34)$ & $(65.30-112.0)$ & $(0.82-1.69)$ & $(1.44-90.50)$ & $(0.06-0.59)$ & $(672.6-1622.7)$ \\
Trans-F2 & $7.16 \pm 0.98$ & $91.0 \pm 25.4$ & $1.32 \pm 0.33$ & $19.18 \pm 18.64$ & $0.39 \pm 0.13$ & $801.1 \pm 88.1$ \\
& $(4.95-8.32)$ & $(40.2-118.6)$ & $(0.94-1.78)$ & $(1.62-84.42)$ & $(0.08-0.61)$ & $(672.6-1659.6)$ \\
$\mathrm{P}$ & $<\mathbf{0 . 0 1}$ & 0.47 & 0.15 & 0.40 & 0.61 & $\mathbf{0 . 0 3}$ \\
\hline
\end{tabular}

Data are expressed as mean \pm SD $(\min -\max )$

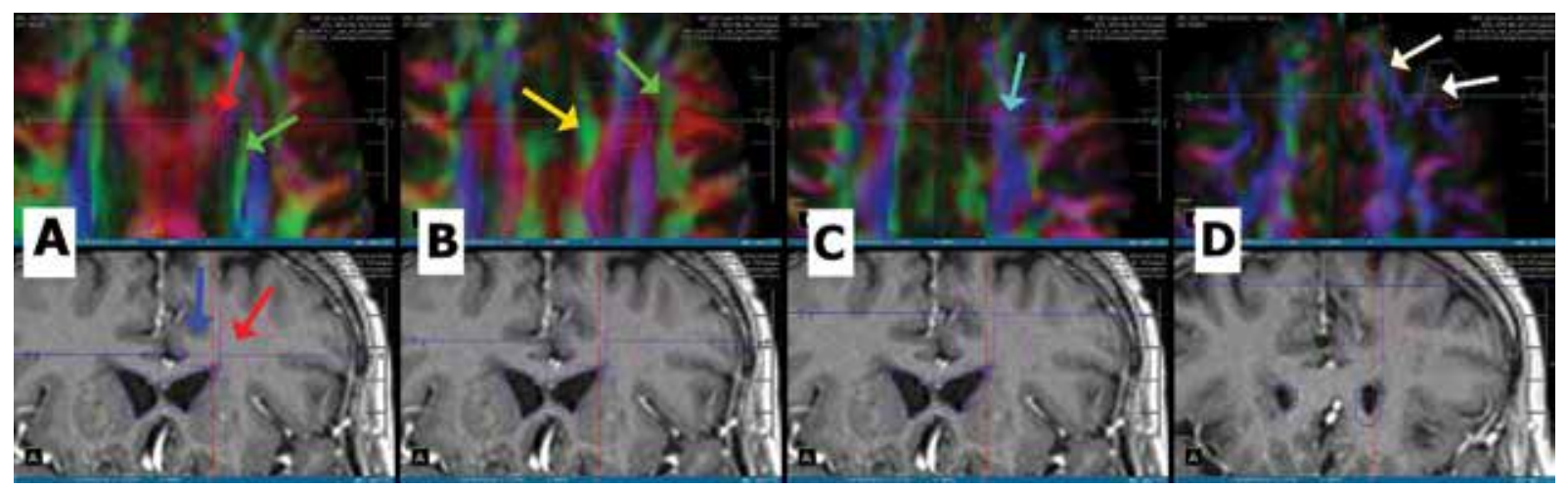

Figure 3. White matter fibres presented in Directionally Encoded Colours (DEC). Two different approaches to lateral ventricle (LV) and foramen of Monro (FM) are indicated: trans-F1 (marked by thin dark purple line in every picture and by blue arrow in lower 3A figure) and trans-F2 (marked by thin light purple line in every picture and with red arrow in upper $3 A$ figure). Top row presents DEC axial planes. The colours of directions are coded with the $X Y Z-R G B$ principal eigenvector colour orientation: red — lateral $\leftrightarrow$ medial; green — rostral $\leftrightarrow$ caudal; blue — ventral $\leftrightarrow$ dorsal. Bottom row visualises T1 coronal planes. Blue thin line in each picture of the bottom row corresponds to the plane of each of the top row DEC axial section. A. Red arrow in DEC axial plane indicates the disruption of callosal fibres caused by trans-F1 and partially by trans-F2 corridor. Superior fronto-occipital fasciculus (not analysed in the current study; marked with green arrow) hypothetically would be cut if trans-F2 approach projected laterally by incidence. Intraoperative use of navigation is to prevent this mistake from happening. B. Superior longitudinal fascicullus I and short association fibres (green arrow) lay in the way of trans-F2 trajectory. There is a hazard of cingulum interruption (yellow arrow) mainly during the access via superior frontal lobe. C. Pyramidal or corticoreticular tract (blue arrow) may be disrupted by both of the frontal transcortical approaches to LV or FM. Only diffusion tensor tractography supports the exact distinction between pyramidal and extrapyramidal tract. D. Cortical diffusion tensor imaging fibres are destroyed by either trans-F1 or trans-F2 approach. The visualisation of white matter by DEC is found useless in terms of selection of the cortical incision area.

Lower number of DTI fibres of SLF I, corpus callosum and cingulum was interrupted by trans-F2 corridor. On the contrary, trans-F1 trajectory was responsible for lesser damage of DTI fibres of SLF II, corticoreticular and pyramidal tract. The significant differences in the extension of fibres' damage between both approaches regarded SLF I and cingulum (the results supported trans-F2 access), as well as corticoreticular tract (trans-F1 was supported) (Table 2).

The analysed material included 2 clinical cases of ventricular tumours. They were operated via trans-F1 approach, followed by planning of trajectory to LV using DTT. Those patients did not present any approach-related complications after the surgery.

Beyond white matter disruption, approaches to LV and FM damage brain cortex. Trans-F1 and trans-F2 requires incision of superior and middle frontal gyrus respectively. In this work, we have observed that cortex within cingulate sulcus (a part of limbic system) might be partially interrupted by trans-F1 corridor. That was noted in $13.6 \%$ of theoretically delineated trajectories starting from superior frontal gyrus (3 of 22). However, shifting the middle boundary of the corridor laterally by at least $4 \mathrm{~mm}$ decreased the risk of violation of frontal midline cortex (Fig. 5). 


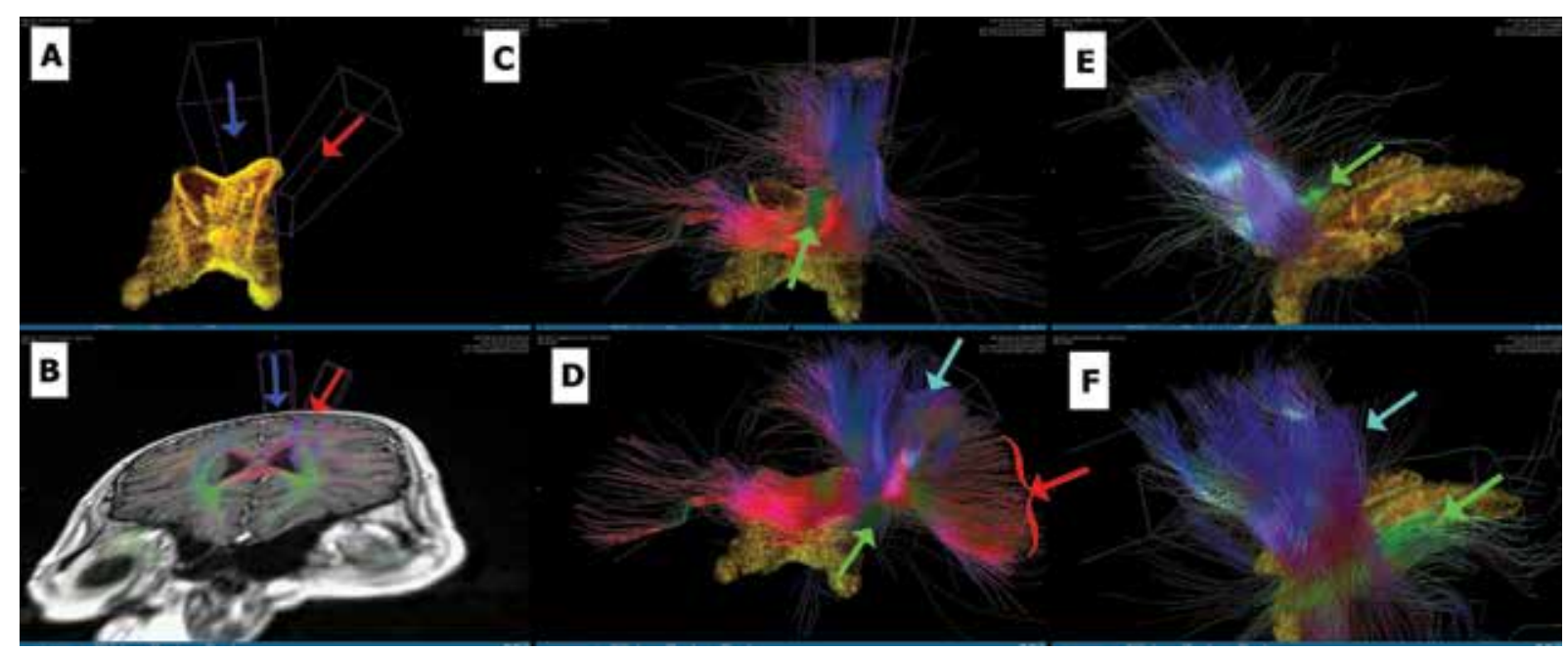

Figure 4. Three-dimensional visualisation of diffusion tensor tractography. Picture $\mathbf{a}$ and $\mathbf{B}$ (left column): trans-F1 and trans-F2 are marked by blue and red arrow respectively. The corridors of both frontal transcortical approaches are outlined by thin purple line. Pictures $\mathbf{C}$ and $\mathbf{E}$ (top): all diffusion tensor imaging (DTI) fibres crossing trans-F1 approach. Pictures $\mathbf{D}$ and $\mathbf{F}$ (bottom): all DTI fibres crossing trans-F2 approach. Pictures $\mathbf{C}$ and $\mathbf{D}$ (middle column): anterior view. Pictures $\mathbf{E}$ and $\mathbf{F}$ (right column): lateral view. A. (Anterior view) lateral ventricles are segmented and coloured yellow. B. Axial plane with both transcortical approaches. T1 magnetic resonance imaging exam and Directionally Encoded Colours are merged, hence the relative position of DTI fibres and anatomical structures is visualised. C. The approach through superior frontal gyrus violates cingulum (green arrow). D. Trans-F2 corridor damages a part of pyramidal/tract (blue arrow), superior longitudinal fascicullus (SLF) I (green arrow) as well as cortical connections with pars triangularis and opercularis of inferior frontal gyrus (red arrow). Extensive projection of the frontal aslant tract (linking superior and inferior frontal gyrus), which might be violated by a lateral aspect of trans-F2 corridor, is marked by red curly bracket. E. Trajectory to lateral ventricle or foramen of Monro through superior frontal gyrus interrupts cingulum (green arrow). F. Partial damage of pyramidal and extrapyramidal tracts (blue arrow). The severing superior part of SLF I (green arrow).

Table 2. Mean quantity of fibres detected by diffusion tensor imaging (DTI) method; neural bundles are specified. The percentages of disrupted fibres of each bundle were compared between 2 frontal transcortical approaches

\begin{tabular}{|c|c|c|c|c|}
\hline \multirow[t]{3}{*}{ Neural bundle } & \multirow{3}{*}{$\begin{array}{c}\text { DTI fibres } \\
\text { Total number } \pm \text { SD (min-max) }\end{array}$} & \multicolumn{3}{|c|}{ Disruption by approach } \\
\hline & & Trans-F1 & $\mathbf{P}$ & Trans-F2 \\
\hline & & \multicolumn{3}{|c|}{ Per cent of disrupted fibres from a neural bundle (number $\pm \mathrm{SD}$; min-max) } \\
\hline SLFI & $28 \pm 9(5-46)$ & $78.6 \%\left(22 \pm 6 ; 10-46^{*}\right)$ & $P<0.001$ & $28.6 \%(8 \pm 9 ; 5-36)$ \\
\hline SLF ॥ & $164 \pm 42(8-221)$ & $15.9 \%(26 \pm 30 ; 8-159)$ & $P=0.051$ & $26.2 \%\left(43 \pm 26 ; 88-221^{*}\right)$ \\
\hline Pyramidal tract & $681 \pm 296(327-1301)$ & $0.6 \%\left(4 \pm 14 ; 0^{\dagger}-60\right)$ & $P=0.210$ & $1.3 \%(9 \pm 12 ; 1-73)$ \\
\hline Corticoreticular tract & $88 \pm 25(45-134)$ & $4.5 \%\left(4 \pm 2 ; 0^{\dagger}-11\right)$ & $P<0.001$ & $30.7 \%(27 \pm 9 ; 3-28)$ \\
\hline Corpus callosum & $973 \pm 108(683-1158)$ & $7.9 \%(77 \pm 22 ; 43-118)$ & $P=0.317$ & $7.3 \%(71 \pm 17 ; 40-115)$ \\
\hline Cingulum & $85 \pm 15(62-109)$ & $49.4 \%(42 \pm 9 ; 27-69)$ & $P<0.001$ & $10.6 \%\left(9 \pm 4 ; 0^{\dagger}-10\right)$ \\
\hline
\end{tabular}

TThere was at least 1 hemisphere, when a specific neural bundle remained intact by the corridor of trans-F1 or trans-F2; "There was at least 1 hemisphere, when a specific neural bundle was totally interrupted by the corridor of trans-F1 or trans-F2; SLF — superior longitudinal fasciculus

\section{DISCUSSION}

Two different routes to LV cause various traumatisation by surgical manipulation: densely packed fibres of corpus callosum are traversed in interhemispheric approach [34], whereas transcortical approach damages the eloquent grey and white matter areas of frontal lobe [50,52]. Some authors believed that post-operative seizure rate is higher when using transcortical approach [6], but others imputed increased seizure occurrence to interhemispheric access [5]. However, the most recent studies provide opposite suggestions [2]. Among transcortical avenues to LV and FM, either the access via superior (trans-F1), middle frontal gyrus (trans-F2) or superior frontal sulcus is also deemed equivalent. Nowadays, the application of emerging techniques, including DTT and its adoption to intraoperative navigation, allows to tailor the craniotomy, minimalise cortical incision 


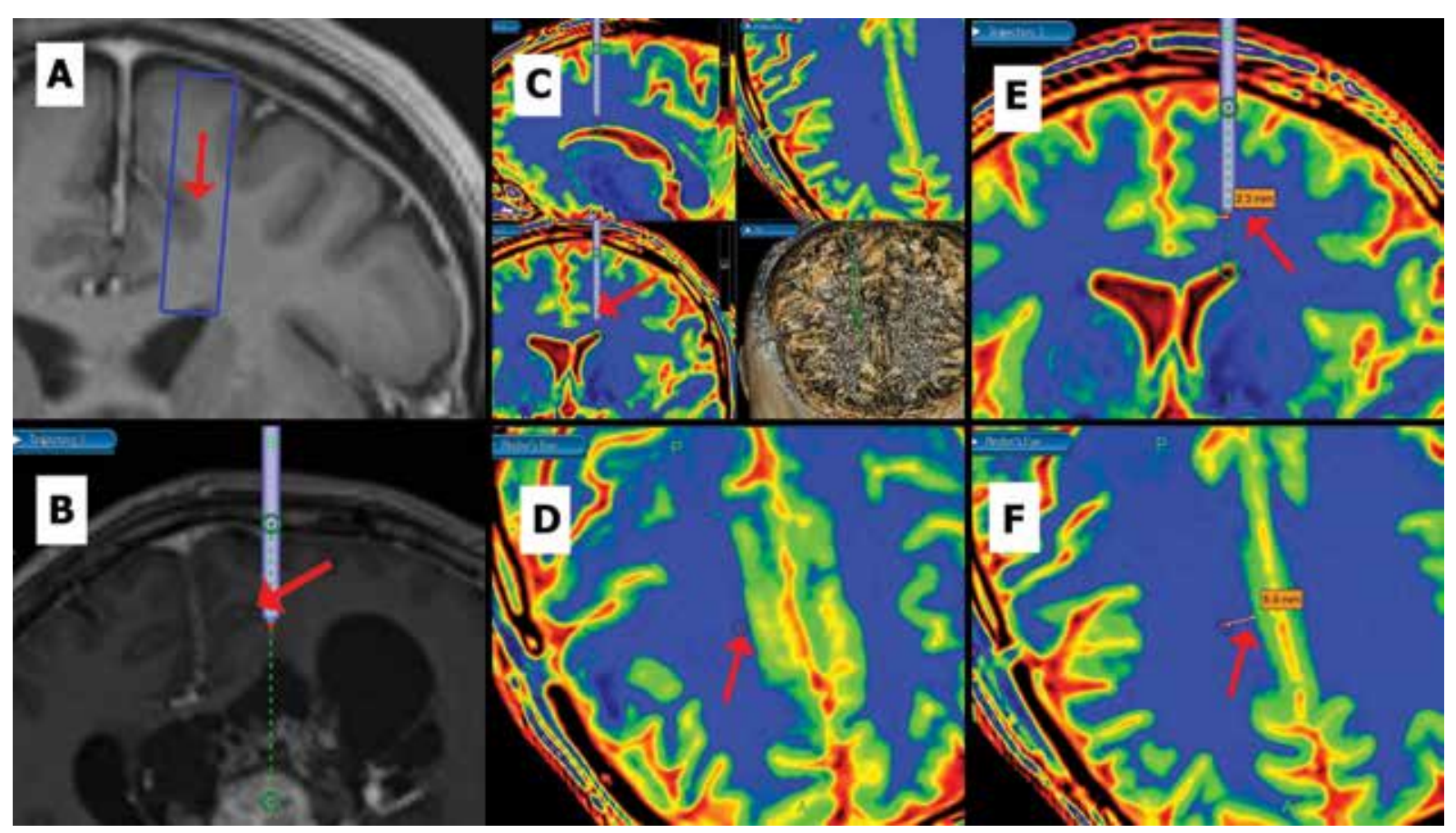

Figure 5. The examples of medial frontal cortex damage caused by trans-F1 trajectory. The trajectory to lateral ventricle (LV) via superior frontal gyrus violates grey matter of cingulate sulcus (marked by red arrow in every picture). A. (T1 magnetic resonance imaging [MRI]; coronal plane) standard, $15 \mathrm{~mm}$ width, hypothetical corridor of trans-F1 approach (marked blue). B. (T1 MRI; coronal plane, modified by trajectory) the patient was operated due to neurocytoma of LV. Coexistence of hydrocephalus theoretically facilitates the approach via medial frontal gyrus. C-F. (T1 MRI; different planes) MRIs are transformed for temperature colour coding. This process allows better distinguishing between white matter (depicted in blue) and grey matter (coded in green) of medial aspect of frontal lobe. D, F. ('probe eye views') — the plane is perpendicular to the trajectory of trans-F2.

as well as assess fibres that are damaged by potential corridor [40,52]. DTI was originally proposed by Basser et al. in $1994[7,8]$. That method was developed from diffusion MRI and has been applied to clinical use and tremendous variety of neuroscientific studies [30]. DTT can be used to explore an individual anatomy and provides the opportunity to stipulate various approaches before the surgery $[52,56]$. However, that technique at present is not used routinely in planning of the ideal trajectory to ventricles. Many years ago Yasargil and Abdulrauf [53] introduced the shift from transcerebral to transventricular access. Their philosophy was to decrease the damage of cortical and subcortical structures. The comparison of trans-F1 and trans-F2 approaches in terms of the extension of white matter disruption has not been discussed so far. Moreover, the quantitive analysis of neural bundles crossing these 2 routes to LV or FM has not been performed either.

In this study, the route through superior frontal gyrus caused significantly greater volume of white matter damage than the access via middle frontal gyrus. However, total fibre track volume, count or length of fibres severed by trans-F1 and trans-F2 were similar. These qualitative data do not reflect potential clinical consequences for the patients. Numerous neural bundles can be at least partially destroyed by microsurgical manipulation during both frontal transcortical approaches, all of which could potentially lead to a surgery-related morbidity [52]. Post-operative complications of any of the transcortical approaches would depend on the specific neural bundle disruption, the damage of which is known to produce unique symptoms. Therefore, the analysis of each neural pathway violated by trans-F1 or trans-F2 working corridor is validated.

The most important motor pathway in the human brain is the pyramidal tract. It arises from the motor cortex and travels through the corona radiata and posterior limb of internal capsule $[26,42]$. The current study have proven, that both trans-F1 and trans-F2 corridors violate only $1 \%$ of upper motor neuron axons, causing theoretically at most minor focal paresis of lower extremity. Relatively high hemiparesis 
rate after the removal of ventricular tumour in clinical series disagrees with the low rate of fibres converging into corona radiata disruption in our study. It might reflect the principal pathogenic role of surgical manipulation within thalamus, basal ganglia, internal capsule or vasogenic oedema in hemiparesis evolvement [34]. Corticobulbar and corticospinal projection fibres run together and are not discriminated on DTT colour maps [28]. Corticoreticular fibres originate mainly from premotor cortex (Brodmann's area 6) and are involved in postural control and locomotor function [33]. The fibres of rostral premotor cortex origin are longer than corticospinal fibres among whole head scan area. It can explain our observation that the longest fibres were severed only by trans-F1 corridor. The virtual incision of middle frontal gyrus in the approach to LV concerned the violation of $1 / 3$ of corticoreticular fibres in significantly greater extension than the access through superior frontal gyrus did. Adversely, significantly lesser damage of 2 association fibres was observed when trans- $\mathrm{F} 2$ approach was applied. Cingulum projects from the cingulate gyrus to the entorhinal cortex, runs arching around the corpus callosum and ensures the communication between the components of the limbic system. That neural bundle integrates disparate systems involved in emotion, attention and memory [16, 29, 35]. It has been used as a target for lesion in psychoneurosurgery, it has also been stimulated in intractable pain syndromes $[11,46]$. In our work, trans-F1 corridor was responsible for the interruption of approximately half of all cingulum fibres. The second route to LV or FM severed only $10 \%$ of that bundle. However, the vulnerability to unilateral anterior cingulotomy is not thoroughly documented. Cognition impairment, personality disruption, akinetic mutism and response inhibition are ascribed to bilateral cingulotomy even in long-term follow-up [21]. Other results confirm that unilateral cingulum disruption in the access to LV results in similar rates of neglecting, memory deficits and naming disturbances as the transcallosal approach [19, 34, 55]. SLF is the major association interhemispheric collection of fibres. Subdivision I of SLF contributes to the regulation of higher aspects of motor behaviour; bidirectional SLF II relates to spatial working memory [17, 27, 32]. In the current study, three quarters of SLF I fibres were interrupted by trans-F1 corridor, nevertheless, in 1 case the whole SLF I bundle was cut. The disruption of SLF I might be unnoticed after the frontal transcortical approach is used, as its clinical significance has not been elucidated [27]. Greater functional role is ascribed to SLF II. Articulate fasciculus, which connects Wernicke's with Broca's area, runs contiguously with SLF II [57]. One third of the patients operated with either transcallosal (far from articulate fasciculus) or frontal transcortical approach are affected by aphasia [34]. In 1 virtual trans-F2 corridor planning from our series, the whole SLF II was cut. Therefore, post-operative speech disturbances cannot be simply explained by the partial damage of SLF II [19]. Corpus callosum was the only commissural fibre pathway violated by the frontal transcortical route. Less than $10 \%$ of all callosal commissure fibres were discovered in the corridor to LV regardless of the route. The transcallosal approach to LV or FM produces higher post-operative seizure rate than the transcortical route $[18,34,49]$. Controversial theories, regarding pathophysiology of seizures after the removal of tumours occupying LV or FM, hold that normal callosal white matter function is implicated in the synchronisation of potential epileptiform foci and thus limits the spread of seizure activity [41]. Equal damage of callosal fibres produced by trans-F1 and trans-F2 proves that similar seizure occurrence might be related to these approaches, although it has not been clinically compared.

FA and $A D C$ are indirect markers of white matter microstructural properties. Both are influenced by numerous factors and both describe a different feature of the fibres [10]. FA indicates the directionality of the diffusion process and reflects the degree of axonal alignment within the voxel of a brain tissue [51]. Hence, it describes fibre density, axonal diameter and myelination in white matter. Furthermore, the mean FA is relatively similar in each structure across patients, yet differs across the structures [38]. ADC is increased in neural bundles that are coherently oriented to gradient direction, in older patients and also in immature brain, regions of oedema or hypoxia [37, 47]. In our material, trans-F2 corridor consisted of slightly more DTI fibres, while ADC was significantly decreased. FA did not vary among frontal transcortical routes. The above-mentioned relations between anisotropy, diffusion and fibre count maintain, that both approaches comprise similar number and density of fibres, but present distinct overall direction of fibres (Fig. 4C-F). Several limitations of the above conclusion result from unexplored scientific value of FA and ADC [12, 25, 37, 51].

There are several limitations of DTT technique. Pitfalls should be consecutively considered in the 
interpretation of fibre tracking results. DTT is strongly dependent on MRI acquisition, patient's movement, the technique of DTI preparation, software, operator, fusion accuracy, exact placement of ROI, employed method of data analysis, etc. [9, 12]. Furthermore, DTT does not reflect small neural pathways and is restricted to regions of non-crossing bundles [54]. It seems highly probable that the use of more precise analytic algorithms, including cross fibres and small neural bundles, would alter the results of that study. However, DTT is a powerful anatomic imaging tool of demonstrating gross fibre architecture, in particular major neural bundles.

\section{CONCLUSIONS}

We have proven that the trans-F2 and trans-F1 approaches cause greater damage of respectively: projection and association fibres. In conclusion, presented results do favour a frontal transcortical route to LV and FM via superior frontal gyrus. SLF II, pyramidal and corticoreticular tracts are more vulnerable when a route starts at the middle frontal gyrus. Hen$\mathrm{ce}$, a violation of these fibres by trans-F2 corridor, even insignificantly greater number than trans- $F 1$ route, is likely to result in a serious disability. Based on DTI findings, the route through superior frontal gyrus is considered more beneficial. However, only a prospective randomised clinical trial concerning these 2 routes would resolve that debate. Lack of contemporary guidelines reflects current decision hesitancy before surgery. A detailed and individual-based preoperative planning, taking advantage of DTI, as well as applying intraoperative neuronavigation, should precede every LV or FM tumour surgery.

\section{ACKNOWLEDGEMENTS}

Special thanks to Agnieszka Sabisz (medical physicist from Radiology Department, Medical University of Gdansk) for the description of magnetic resonance imaging acquisition parameters, Marta Adamska for preparing Figure 1 and Agnieszka Prylińska for grammar and vocabulary improvements.

\section{REFERENCES}

1. Abe O, Masutani Y, Aoki S, Yamasue H, Yamada H, Kasai $K$, Mori H, Hayashi N, Masumoto T, Ohtomo K (2004) Topography of the human corpus callosum using diffusion tensor tractography. J Comput Assist Tomogr, 28: 533-539.

2. Aicardi J (2008) Epilepsy syndromes. In: Engel J, Pedley T eds. Epilepsy: a comprehensive textbook. Lippincott Williams \& Wilkins, New York, pp. 2283-2695.
3. Antunes JL, Louis KM, Ganti SR (1980) Colloid cysts of the third ventricle. Neurosurgery, 7: 450-455.

4. Apuzzo ML (1988) Surgery of masses affecting the third ventricular chamber: techniques and strategies. Clin Neurosurg, 34: 499-522.

5. Aryan HE, Ozgur BM, Jandial R, Levy ML (2006) Complications of interhemispheric transcallosal approach in children: review of 15 years experience. Clin Neurol Neurosurg, 108: 790-793.

6. Asgari S, Engelhorn T, Brondics A, Sandalcioglu IE, Stolke D (2003) 'Transcortical or transcallosal approach to ventricle-associated lesions: a clinical study on the prognostic role of surgical approach. Neurosurg Rev, 26: 192-197.

7. Basser PJ, Mattiello J, LeBihan D (1994) Estimation of the effective self-diffusion tensor from the NMR spin echo. J Magn Reson B, 103: 247-254.

8. Basser PJ, Mattiello J, LeBihan D (1994) MR diffusion tensor spectroscopy and imaging. Biophys J, 66: 259-267.

9. Basser PJ, Pajevic S, Pierpaoli C, Duda J, Aldroubi A (2000) In vivo fiber tractography using DT-MRI data. Magn Reson Med, 44: 625-632.

10. Beaulieu C (2002) The basis of anisotropic water diffusion in the nervous system: a technical review. NMR Biomed, 15: 435-455.

11. Broggi G (2008) Pain and psycho-affective disorders. Neurosurgery, 62: 901-919 (discussion 919-920).

12. Bull JG (2010) Diffusion imaging and tractography. In: The paediatric neurosurgical population. University College, London.

13. Cairns H (1971) Who's in charge here?. Lancet, 2: 318-319.

14. Carrasco EM (2011) Left intraventricular tumour resected via superior frontal transulcal transcortical approach. Neurosurgic blog and news channel. NEUROSURGIC.com, Santa Cruz, Bolivia.

15. Casmiro $M$, Sintini $M$, Martinelli $P$, Leonardi $M$, D'alessandro $R$ (1992) Chronic spastic atrophic hemiparesis with benign course. Ital J Neurol Sci, 13: 611-616.

16. Catani M, Dell'acqua F, Vergani F, Malik F, Hodge H, Roy P, Valabregue R, Thiebaut De Schotten M (2012) Short frontal lobe connections of the human brain. Cortex, 48 : 273-291.

17. Catani M, Howard RJ, Pajevic S, Jones DK (2002) Virtual in vivo interactive dissection of white matter fasciculi in the human brain. Neuroimage, 17: 77-94.

18. Ellenbogen RG (2001) Transcortical surgery for lateral ventricular tumors. Neurosurg Focus, 10: E2.

19. Friedman MA, Meyers CA, Sawaya R (2003) Neuropsychological effects of third ventricle tumor surgery. Neurosurgery, 52: 791-798 (discussion 798).

20. Fuh JL, Wang SJ (1995) Caudate hemorrhage: clinical features, neuropsychological assessments and radiological findings. Clin Neurol Neurosurg, 97: 296-299.

21. Gasquoine PG (2013) Localization of function in anterior cingulate cortex: from psychosurgery to functional neuroimaging. Neurosci Biobehav Rev, 37: 340-348.

22. Hasan KM, Kamali A, Iftikhar A, Kramer LA, Papanicolaou AC, Fletcher JM, Ewing-Cobbs L (2009) Diffusion tensor tractography quantification of the human corpus callosum fiber pathways across the lifespan. Brain Res, 1249: 91-100.

23. Hassaneen W, Suki D, Salaskar AL, Levine NB, Demonte F, Lang FF, Mccutcheon IE, Dorai Z, Feiz-Erfan I, Wildrick DM, Sawaya R (2010) Immediate morbidity and mortality associated with transcallosal resection of tumors of the third ventricle. J Clin Neurosci, 17: 830-836.

24. Hernesniemi J, Romani R, R. Dashti, Albayrak BS, Savolainen S, Ramsey C, Karatas A, Lehto H, Navratil O, Niemelä M (2008) Microsurgical treatment of third ventricular colloid 
cysts by interhemispheric far lateral transcallosal approach: experience of 134 patients. Surg Neurol, 69: 447-453 (discussion 453-446).

25. Hoeft F, Barnea-Goraly N, Haas BW, Golarai G, Ng D, Mills D, Korenberg J, Bellugi U, Galaburda A, Reiss AL (2007) More is not always better: increased fractional anisotropy of superior longitudinal fasciculus associated with poor visuospatial abilities in Williams syndrome. J Neurosci, 27: 11960-11965.

26. Ino T, Nakai R, Azuma T, Yamamoto T, Tsutsumi S, Fukuyama H (2007) Somatotopy of corticospinal tract in the internal capsule shown by functional MRI and diffusion tensor images. Neuroreport, 18: 665-668.

27. Jang SH, Hong JH (2012) The anatomical characteristics of superior longitudinal fasciculus I in human brain: Diffusion tensor tractography study. Neurosci Lett, 506: 146-148.

28. Jellison BJ, Field AS, Medow J, Lazar M, Salamat MS, Alexander AL (2004) Diffusion tensor imaging of cerebral white matter: a pictorial review of physics, fiber tract anatomy, and tumor imaging patterns. Am J Neuroradiol, 25: 356-369.

29. Keedwell PA, Chapman R, Christiansen K, Richardson H, Evans J, Jones DK (2012) Cingulum white matter in young women at risk of depression: the effect of family history and anhedonia. Biol Psychiatry, 72: 296-302.

30. LeBihan D, Mangin JF, Poupon C, Clark CA, Pappata S, Molko N, Chabriat H (2001) Diffusion tensor imaging: concepts and applications. J Magn Reson Imaging, 13: 534-546.

31. Lin F, Yu C, Jiang T, Li K, Li X, Qin W, Sun H, Chan P (2006) Quantitative analysis along the pyramidal tract by length-normalized parameterization based on diffusion tensor tractography: application to patients with relapsing neuromyelitis optica. Neuroimage, 33: 154-160.

32. Makris N, Kennedy DN, Mcinerney S, Sorensen AG, Wang R, Caviness VS, Pandya DN (2005) Segmentation of subcomponents within the superior longitudinal fascicle in humans: a quantitative, in vivo, DT-MRI study. Cereb Cortex, 15: 854-869.

33. Matsuyama K, Mori F, Nakajima K, Drew T, Aoki M, Mori S (2004) Locomotor role of the corticoreticular-reticulospinal-spinal interneuronal system. Prog Brain Res, 143: 239-249.

34. Milligan BD, Meyer FB (2010) Morbidity of transcallosal and transcortical approaches to lesions in and around the lateral and third ventricles: a single-institution experience. Neurosurgery, 67: 1483-1496 (discussion 1496).

35. Morgane PJ, Galler JR, Mokler DJ (2005) A review of systems and networks of the limbic forebrain/limbic midbrain. Prog Neurobiol, 75: 143-160.

36. Moore AJ, Newell DW (2005) Neurosurgery: principles and practice. Springer, London.

37. Mukherjee P, Berman JI, Chung SW, Hess CP, Henry RG (2008) Diffusion tensor MR imaging and fiber tractography: theoretic underpinnings. Am J Neuroradiol, 29: 632-641.

38. O'donnell L, Haker S, Westin C (2002) New approaches to estimation of white matter connectivity in diffusion tensor MRI: Elliptic PDE's and geodesics in tensor-warped space. In: Proc. of Medical Image Computing and Computer-Assisted Intervention (MICCAI), Springer, Berlin Heidelberg, pp. 459-466.

39. Park ES, Cho YH, Kim JH, Kim SJ, Khang SK, Kim CJ (2012) Frontal transcortical approach in 12 central neurocytomas. Acta Neurochir, 154: 1961-1971 (discussion 1972).

40. Perneczky A, Reisch R (2008) Transcortical approach. Keyhole approaches in neurosurgery. Vol. 1: Concept and surgical technique. Springer-Verlag, Wien, pp. 267-282.
41. Prince DA (1985) Physiological mechanisms of focal epileptogenesis. Epilepsia, 26 (suppl. 1): S3-S14.

42. Qazi AA, Radmanesh A, O'donnell L, Kindlmann G, Peled S, Whalen S, Westin CF, Golby AJ (2009) Resolving crossings in the corticospinal tract by two-tensor streamline tractography: Method and clinical assessment using fMRI. Neuroimage, 47: Suppl 2 T98-106.

43. Raj A, Kuceyeski A, Weiner M (2012) A network diffusion model of disease progression in dementia. Neuron, 73: 1204-1215.

44. Shinoura N, Onodera T, Kurokawa K, Tsukada M, Yamada R, Tabei Y, Koizumi T, Yagi K (2010) Damage to the upper portion of area 19 and the deep white matter in the left inferior parietal lobe, including the superior longitudinal fasciculus, results in alexia with agraphia. Eur Neurol, 64: 224-229.

45. Shinoura N, Suzuki Y, Yamada R, Tabei Y, Saito K, Yagi K (2009) Damage to the right superior longitudinal fasciculus in the inferior parietal lobe plays a role in spatial neglect. Neuropsychologia, 47: 2600-2603.

46. Spooner J, Yu H, Kao C, Sillay K, Konrad P (2007) Neuromodulation of the cingulum for neuropathic pain after spinal cord injury. Case report. J Neurosurg, 107: 169-172.

47. Sullivan EV, Adalsteinsson E, Pfefferbaum A (2006) Selective age-related degradation of anterior callosal fiber bundles quantified in vivo with fiber tracking. Cereb Cortex, 16: 1030-1039.

48. Vigneron LM, Warfield SK, Robe PA, Verly JG (2011) 3D XFEM-based modeling of retraction for preoperative image update. Comput Aided Surg, 16: 121-134.

49. Villani R, Tomei $G$ (2006) Approach to tumors of the third ventricle. In: Schmidek H, Roberts D eds. Schmidek and Sweet's operative neurosurgical techniques: indications, methods, and results. Saunders Elsevier, Philadelphia, pp. 772-785.

50. Wen HT, Mussi ACM, Rhoton AL Jr., Oliveira ED, Tedeschi H (2006) Surgical approaches to lesions located in the lateral, third, and fourth ventricles. In: Sekhar LN, Fessler RG eds. Atlas of neurosurgical techniques: brain. Thieme, New York, pp. 527-528.

51. Westerhausen R, Huster RJ, Kreuder F, Wittling W, Schweiger $E$ (2007) Corticospinal tract asymmetries at the level of the internal capsule: is there an association with handedness? Neuroimage, 37: 379-386.

52. Winn HR (2011) Youmans neurological surgery. Elsevier Saunders, Philadelphia.

53. Yaşargil MG, Abdulrauf SI (2008) Surgery of intraventricular tumors. Neurosurgery, 62: 1029-1040 (discussion 1040-1021).

54. Yeo SS, Chang MC, Kwon YH, Jung YJ, Jang SH (2012) Corticoreticular pathway in the human brain: diffusion tensor tractography study. Neurosci Lett, 508: 9-12.

55. Yonekawa T, Nakagawa E, Takeshita E, Inoue Y, Inagaki M, Kaga M, Sugai K, Sasaki M, Kaido T, Takahashi A, Otsuki T (2011) Effect of corpus callosotomy on attention deficit and behavioral problems in pediatric patients with intractable epilepsy. Epilepsy Behav, 22: 697-704.

56. Yu CS, Li KC, Xuan Y, Ji XM, Qin W (2005) Diffusion tensor tractography in patients with cerebral tumors: a helpful technique for neurosurgical planning and postoperative assessment. Eur J Radiol, 56: 197-204.

57. Zhao $Y$, Chen X, Wang F, Sun G, Wang Y, Song Z, Xu B (2012) Integration of diffusion tensor-based arcuate fasciculus fibre navigation and intraoperative MRI into glioma surgery. J Clin Neurosci, 19: 255-261. 